\title{
Editorial für die Zeitschrift für Orthopädie und Unfallchirurgie
}

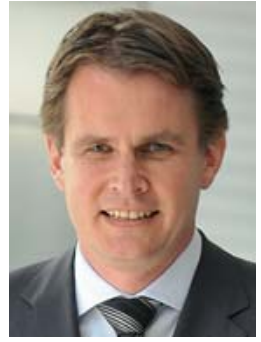

U. Stöckle

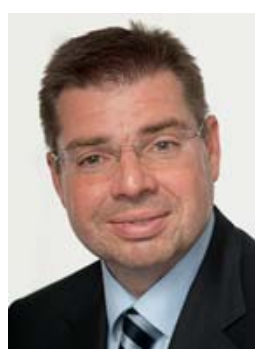

D. C. Wirtz

\author{
Liebe Leserinnen, liebe Leser, \\ $\nabla$
}

mit der aktuellen Ausgabe der Zeitschrift für Orthopädie und Unfallchirurgie haben wir wieder versucht, aktuelle Themen aus dem Bereich der Orthopädie und Unfallchirurgie mit den unterschiedlichen Veröffentlichungsformaten abzubilden. Thematischer Schwerpunkt ist hierbei die Endoprothetik. Die aktuell vielfach diskutierte Bedeutung und der mögliche Vorteil minimalinvasiver Zugangswege in der primären Hüftendoprothetik werden in einem ausführlichen Reviewartikel untersucht. Bei aktuell nur wenig randomisiert kontrollierten Studien und sich offensichtlich nach etwa 12 Monaten postoperativ angleichenden Werten für die Funktion des operierten Gelenkes lässt sich aktuell noch kein eindeutiger Vorteil der minimalinvasiven Zugangswege darstellen. Dies zeigt deutlich den Bedarf weiterer randomisiert kontrollierter Studien mit größerer Fallzahl, um den Vorteil innovativer Operationsverfahren darstellen zu können, sodass aus einer Innovation auch wirklich Fortschritt für den Patienten werden kann. Die Implantation einer Hüfttotalendoprothese über einen minimalinvasiven Zugang wird auch mit unserem neuesten Medium, dem Videopaper dargestellt. Diese im letzten Jahr eingeführte Form der Veröffentlichung in Zusammenarbeit mit der Deutschen Gesellschaft für Chirurgie und der AG Medien erweist sich als sehr hilfreiches und gut angenommenes Medium zur Darstellung aktueller und insbesondere auch neuer innovativer Operationsverfahren. Neben einer Originalarbeit zum Einfluss der Kapselrekonstruktion auf die Luxationsrate nach primärer Hüftendoprothetik anhand einer retrospektiven Analyse stellt schließlich eine Arbeit aus der Arbeitsgemeinschaft für Endoprothetik die Datenlage zum patellofemoralen Ersatz dar. Die Originalarbeiten sind natürlich das „Herzstück“ einer wissenschaftlichen Zeitschrift wie der Zeitschrift für Orthopädie und Unfallchirurgie. Wir sehen es daneben aber auch als unsere Aufgabe als offizielles Organ der Deutschen Gesellschaft für Orthopädie und Unfallchirurgie, qualitativ hochwertige Beiträge aus den Arbeitsgemeinschaften und Sektionen nach entsprechendem Reviewverfahren zu veröffentlichen. Dies ist am Beispiel der Arbeitsgemeinschaft für Endoprothetik exemplarisch inzwischen erfolgreich etabliert. Wie in der vorliegenden aktuellen Ausgabe für die Endoprothetik bilden die unterschiedlichen Formate des Originalartikels, Reviewartikels, Videopaper und der qualitativ hochwertigen Beiträge aus Arbeitsgemeinschaften und Sektionen den wissenschaftlichen Teil unserer Zeitschrift für Orthopädie und Unfallchirurgie.

Weitere Themen sind schließlich in der vorliegenden Ausgabe die Knochenheilung bzw. Knochenersatz sowie Osteoporose und thromboembolische Komplikation. Entscheidend ist hierbei wiederum, dass aktuelle, innovative Therapieformen wie der Einsatz von BMP kritisch hinterfragt werden und insbesondere versucht wird, aufgrund experimenteller Ergebnisse auch Therapieversager zu erklären und zukünftig die Wirksamkeit solcher Verfahren zu verbessern.

Wir hoffen mit dieser thematischen Mischung und insbesondere auch der angesprochenen unterschiedlichen Formate wie Originalartikel, Reviewartikel, Videopaper und hochqualifizierte Beiträge aus Arbeitsgemeinschaften und Sektionen die Positionierung der Zeitschrift für Orthopädie und Unfallchirurgie als erfolgreiches deutschsprachiges wissenschaftliches Journal im Bereich Orthopädie und Unfallchirurgie festigen und weiter ausbauen zu können.

Ihre

Prof. Dr. med. Ulrich Stöckle

Prof. Dr. med. Dieter C. Wirtz
Univ.-Prof. Dr. Ulrich
Ärztlicher Direktor

BGU Klinik Tübingen

Schnarrenbergstraße 96

72076 Tübingen

Tel.: $07071 / 606-1001$

Fax: 07071/606-1002

ustoeckle@bgu-tuebingen.de

\section{Univ.-Prof. Dr. med.}

Dieter C. Wirtz

Direktor der Klinik und

Poliklinik für Orthopädie

und Unfallchirurgie

Universitätsklinikum Bonn

Sigmund-Freud-Straße 25

53105 Bonn

Tel.: 02 28/2 87-14170

Fax: 0228/287-14175

dieter.wirtz@ukb.uni-bonn.de 